NBI-HE-95-16

hep-th/9504127

April 1995

\title{
CONFORMAL BLOCKS FOR ADMISSIBLE REPRESENTATIONS IN $S L(2)$ CURRENT ALGEBRA
}

\author{
Jens Lyng Petersen凹, Jørgen Rasmussen \ and Ming Yu円 \\ The Niels Bohr Institute, Blegdamsvej 17, DK-2100 Copenhagen Ø, Denmark
}

\begin{abstract}
Despite considerable work in the literature on $N$-point correlators in 2-d conformal WZNW models based on affine $\widehat{S L}(2)_{k}$, either by using the Wakimoto construction or by directly solving the Knizhnik-Zamolodchikov equations, most published results pertain to integrable representations with $t=k+2$ integer and all primary fields having integer or half integer spin. Results for admissible representations corresponding to $t=k+2=$ $p / q$ rational, appear to be rather incomplete, despite their potential interest in various connections, notably in connections to non-critical string theory via $2-d$ gravity based on hamiltonian reductions. Indeed, surprisingly, even the fusion rules remain a subject of discussion. The reason for this state of affairs may be traced to the need in the free field Wakimoto construction for introducing a second screening charge as discussed by Bershadsky and Ooguri, one which depends on fractional powers of free fields, and such entities have until now eluded a consistent interpretation in terms of Wick contractions. In this paper we develop the techniques necessary to deal with these complications, and we provide explicit general integral representations for conformal blocks on the sphere. They turn out to have the structure expected from the operator formalism and one, which renders their consistency check straight forward. We further discuss fusion rules, and as a check we verify explicitly that our conformal blocks satisfy the Knizhnik-Zamolodchikov equations and are projectively invariant.
\end{abstract}

\footnotetext{
${ }^{1}$ e-mail address: jenslyng@nbivax.nbi.dk

${ }^{2}$ e-mail address: jrasmussen@nbivax.nbi.dk

${ }^{3}$ e-mail address: yuming@nbivax.nbi.dk. Address after 1st October 1995: Inst. of Theor. Phys., Academia Sinica, Beijing, Peoples Republic of China
} 


\section{Introduction}

$N$-point correlators of 2 - $d$ conformal WZNW theories based on affine $\widehat{S L}(2)_{k}$, with $k=$ level, have been much studied already. They are typically constructed, either by applying the free field realization of Wakimoto [1], from which results have been given for example in refs. [2, 3, 4, 5], or by solving the Knizhnik-Zamolodchikov equations [6], from which results have been given for example in refs. [6, 7, 8, 9, 10]. Recently the structure of solutions of the Knizhnik-Zamolodchikov equations on higher genus Riemann surfaces has been reexamined [11]. The results given in these various pieces of works are quite complete as far as unitary, integrable representations [12 are concerned, but appear surprisingly incomplete for the general case, including admissible representations. Partial and conjectural results not based on the free field realization were given in [4]. In particular these authors made the interesting conjecture, that minimal model conformal blocks are obtained from the affine $S L(2)$ blocks by a simple substitution, one of identifying the $x_{i}$-variables related to the $i$ 'th $S L(2)$ representation (and to be introduced below), with the Koba-Nielsen variables, $z_{i}$. Based on this conjecture an attempt was made to solve the Knizhnik-Zamolodchikov equations in a power series of $\left(x_{i}-z_{i}\right)$. In contrast we shall find complete integral expressions based on the free field realization and providing exact solutions to the $\mathrm{KZ}$ equations. Using that realization, the conjecture may now be addressed. In this paper we have merely checked it's validity in a few examples. We intend to come back to a detailed discussion of this and related issues of hamiltonian reduction elsewhere.

In general the WZNW theory is characterized by the level, $k$, or equivalently by $t=$ $k+2$ (for $\left.\widehat{S L}(2)_{k}\right)$. Then degenerate primary fields exist for representations characterized by spins, $j_{r s}$, given by [13, 14]

$$
2 j_{r s}+1=r-s t
$$

with $r, s$ integers. However, previous results can be characterized as pertaining only to the special case, $s=0$, which is the full case only for integrable representations. The reason for this restriction is fairly natural, since (see sect. 2) the screening charge usually employed in the free field realization is capable of screening just such primary fields. In fact, a possible second screening operator, capable of screening the general case was proposed by Bershadsky and Ooguri [15], but since it involved fractional powers of the free ghost fields, it apparently has remained unknown how to make use of that screening operator.

In the present paper we overcome this difficulty by showing how the techniques of fractional calculus [16] (briefly described in an appendix) naturally provides a solution. As a result we are able to present general integral formulas for the $N$-point conformal blocks on a sphere, and by using standard sewing techniques it should be possible to generalize those to an arbitrary Riemann surface. Our own motivation lies in our wish to make use of these results to cover the case of admissible representations [17] corresponding to rational values of $t$, since these are the ones relevant for treating conformal minimal matter using hamiltonian reduction [15 coupled to 2- $d$ gravity along the lines of [18, 19]. Once this technique is worked out it would be interesting to generalize to higher groups and supergroups in order to be able to treat more general non-critical string theory. The technique we present here appears directly amenable of such generalizations. 
In sect. 2 we define our notation and introduce the relation to fractional calculus. In sect. 3 we show how to obtain the three point function and we derive fusion rules for admissible representations and compare with results already in the literature. In sect. 4 we derive the $N$-point function and make comments on comparison with known results. In sect. 5 we provide examples concerning 4-point functions. In sect. 6 we prove that the $N$-point functions satisfy the Knizhnik-Zamolodchikov equations. It appears from comparing with known solutions in the mathematics literature [10], that our formulas involving auxiliary integrations represent fairly powerful ways of dealing with such solutions. In fact, the structure of our blocks corresponds very closely to the operator formalism, and any results worked out in the latter can be verified in the former. In sect. 7 we discuss the slightly non trivial way in which projective and global $s l_{2}$ invariance of the correlators is established. In sect. 8 we give a summary and an outlook. In two appendices we briefly describe fractional calculus [16, and we prove some non trivial consistency conditions for the rules for Wick contractions we have derived based on fractional calculus.

\section{Notation for free field realization. Relation to frac- tional calculus}

The Wakimoto realization [1] is based on the free scalar field, $\varphi(z)$, and bosonic ghost fields, $(\beta(z), \gamma(z))$, of dimensions $(1,0)$ which we take to have the following contractions

$$
\varphi(z) \varphi(w)=\log (z-w), \quad \beta(z) \gamma(w)=\frac{1}{z-w}
$$

We only consider one chirality of the fields. The $\widehat{S L}(2)_{k}$ affine currents may then be represented as

$$
\begin{aligned}
J^{+}(z) & =\beta(z) \\
J^{3}(z) & =-: \gamma \beta:(z)-\sqrt{t / 2} \partial \varphi(z) \\
J^{-}(z) & =-: \gamma^{2} \beta:(z)+k \partial \gamma(z)-\sqrt{2 t} \gamma \partial \varphi(z) \\
t & \equiv k+2 \neq 0
\end{aligned}
$$

They satisfy

$$
\begin{aligned}
J^{+}(z) J^{-}(w) & =\frac{2}{z-w} J^{3}(w)+\frac{k}{(z-w)^{2}} \\
J^{3}(z) J^{ \pm}(w) & = \pm \frac{1}{z-w} J^{ \pm}(w) \\
J^{3}(z) J^{3}(w) & =\frac{k / 2}{(z-w)^{2}}
\end{aligned}
$$

The Sugawara energy momentum tensor is obtained as

$$
T(z)=: \beta \partial \gamma:(z)+\frac{1}{2}: \partial \varphi \partial \varphi:(z)+\frac{1}{\sqrt{2 t}} \partial^{2} \varphi(z)
$$


with central charge

$$
c=\frac{3 k}{k+2}
$$

Since we shall be dealing with nonunitary representations, $j$, of $S L(2)$ for which the weight, $m$, may assume infinitely many (integrally spaced) values, it is most convenient to collect the multiplets of primary fields as [0]

$$
\phi_{j}(z, x)=\sum_{m} \phi_{j}^{m}(z) x^{j-m}
$$

Such a primary field satisfies the following OPE's

$$
J^{a}(z) \phi_{j}(w, x)=\frac{1}{z-w} J_{0}^{a}(w) \phi_{j}(w, x)
$$

where the $S L(2)$ representation is provided by the differential operators

$$
\begin{aligned}
J_{0}^{a}(z) \phi_{j}(z, x) & =\left[J_{0}^{a}, \phi_{j}(z, x)\right]=D_{x}^{a} \phi_{j}(z, x) \\
D_{x}^{+} & =-x^{2} \partial_{x}+2 x j \\
D_{x}^{3} & =-x \partial_{x}+j \\
D_{x}^{-} & =\partial_{x}
\end{aligned}
$$

The primary field $\phi_{j}(w, x)$ defined in this way transforms covariantly under both conformal transformations and loop projective transformations, namely as an $h$ tensor field for the former, and $\mathrm{a}-j$ tensor field for the latter,

$$
\begin{aligned}
z & \rightarrow f(z) \\
x & \rightarrow \frac{a(z) x+b(z)}{c(z) x+d(z)}
\end{aligned}
$$

with $a(z) d(z)-b(z) c(z)=1$. One easily verifies that the free field realization of the primary field may be taken as [4]

$$
\phi_{j}(z, x)=(1+\gamma(z) x)^{2 j}: e^{-j \sqrt{2 / t} \varphi(z)}:
$$

where, in general one should asymptotically expand $(1+\gamma(z) x)^{2 j}$ as

$$
(1+\gamma(z) x)_{(\alpha)}^{2 j}=\sum_{n \in \mathbb{Z}}\left(\begin{array}{c}
2 j \\
n+\alpha
\end{array}\right)(\gamma(z) x)^{n+\alpha}
$$

Here, the choice of the parameter $\alpha$ depends on the monodromy conditions of the primary field $\phi_{j}(z, x)$ around contours in $x$-space, and those in turn depend on the other fields present in the correlator.

Let us add various remarks of a general nature. For further details on fractional calculus we refer to Appendix A. The most important rule for us will simply be

$$
\partial_{x}^{a} x^{b}=\frac{\Gamma(b+1)}{\Gamma(b-a+1)} x^{b-a}
$$


Next, consider the fractional derivative of the exponential function:

$$
D^{a} \exp (x)=\sum_{n \in \mathbb{Z}} \frac{1}{\Gamma(n-a+1)} x^{n-a}, \quad a \in \mathbb{C}
$$

This represents a peculiar realization of the exponential function itself, which converges asymptotically for $|x| \rightarrow \infty$. Formally the right hand side is invariant under further differentiation corresponding to the fact that it represents the original exponential function. The representation may be better understood by writing

$$
\exp \{x\}=x^{-a}\left[\frac{e^{x}}{x^{-a}}\right]
$$

and then introducing for the last bracket a Fourier expansion with integer powers of $x$ on a circle in the complex $x$-plane. On the circle

$$
\left[e^{x} x^{a}\right]
$$

has a discontinuity which we may take to be for negative $x$. The Fourier expansion converges for large $|x|$ where the discontinuity becomes vanishingly small. Thus we take

$$
D^{a} \exp (x)=\exp (x)
$$

for any $a$. However, we shall find it convenient to use the fractional derivative to represent a generating functional for the integrals

$$
\lim _{R \rightarrow \infty} \oint_{R S^{1}} \frac{d u}{2 \pi i} \frac{e^{u}}{u^{a+1+n}}=\frac{1}{\Gamma(a+1+n)}
$$

Different $a$ 's give rise to representations or expansions of the exponential function in which individual terms have different non-trivial monodromies.

Similarly, since we shall need contractions with the operator

$$
(1+\gamma(z) x)^{2 j}
$$

we shall find it convenient to represent the expansion of the associated analytic function

$$
f(z) \equiv(1+z)^{2 j}
$$

in several different ways. Indeed, from

$$
(1+z)^{2 j}=z^{a}\left[(1+z)^{2 j} z^{-a}\right]
$$

we may imagine that the last bracket is expanded in integer powers of $z$ in a way convergent on the unit circle (with suitable conditions on $a$ ) since in fact this time the discontinuity is vanishing. This renders many equivalent representation for the function:

$$
(1+z)^{2 j}=\sum_{n \in \mathbb{Z}}\left(\begin{array}{c}
2 j \\
n-a
\end{array}\right) z^{n-a}
$$


which are all equivalent in the sense of analytic function theory but which correspond to expansions with different monodromies for the individual terms.

When deciding on what expansion to adopt for the operator

$$
(1+\gamma(z) x)^{2 j}
$$

we use the criterion, that after all Wick contractions have been performed, powers of $\beta$ and $\gamma$ inside normal ordering signs are non-negative integers, such as was illustrated above. Only then are these terms having an obvious interpretation when sandwiched between states. In other terms, the existence of external states and other primary fields in the correlator decides what monodromies to choose for individual terms in expansions. All of that will be illustrated further below.

To calculate operator product expansion (OPE) of expressions involving the $\beta(z)$ and $\gamma(w)$ fields, when either $\beta$ or $\gamma$ fields appear with integral powers, it is clear that the following rules apply:

$$
\begin{aligned}
& \beta(z)^{n} F(\gamma(w))=:\left(\beta(z)+\frac{1}{z-w} \partial_{\gamma(w)}\right)^{n} F(\gamma(w)): \\
& \gamma(z)^{n} F(\beta(w))=:\left(\gamma(z)-\frac{1}{z-w} \partial_{\beta(w)}\right)^{n} F(\beta(w)):
\end{aligned}
$$

The two relevant screening charge currents are [15]

$$
\begin{aligned}
& S_{1}(z)=\beta(z) e^{+\sqrt{2 / t} \varphi(z)} \\
& S_{2}(z)=\beta(z)^{-t} e^{-\sqrt{2 t} \varphi(z)}
\end{aligned}
$$

From now on we mostly leave out normal ordering signs around exponentials. The screening currents are easily seen to have OPE's with the affine currents that are either nonsingular or form total derivatives (in particular this will follow from our definitions of Wick contractions for fractional powers of free fields below).

As we have seen, the $\gamma(z)$ field can be fractionally powered in asymptotic expansions of the primary field $\phi_{j}(z, x)$. Thus we need insertions of the second screening current $S_{2}(z)$ in the correlators to neutralize those. In such cases, the $\beta$ field has nontrivial monodromy with respect to the primary field $\phi_{j}(z, x)$. Hence our proposal to deal with the awkward looking field,

$$
\beta(z)^{-t}
$$

consists in generalizing eq.(18) as

$$
G(\beta(z)) F(\gamma(w))=: G\left(\beta(z)+\frac{1}{z-w} \partial_{\gamma(w)}\right) F(\gamma(w)):
$$

where, the asymptotic expansions for $G\left(\beta(z)+\frac{1}{z-w} \partial_{\gamma(w)}\right)$ and $F(\gamma(w))$ would depend on their monodromy conditions in the $z$ and $w$ variables respectively. When applying eq.(20) to our particular case, where $F(\gamma)=(1+x \gamma)^{2 j}$, we have

$$
\begin{aligned}
\beta(z)^{-t} F(\gamma(w)) & =:\left(\beta(z)+\frac{1}{z-w} \partial_{\gamma(w)}\right)^{-t} F(\gamma(w)): \\
& =\sum_{n=0}^{\infty}\left(\begin{array}{c}
-t \\
n
\end{array}\right): \beta^{n}(z)(z-w)^{t+n} \partial_{\gamma(w)}^{-t-n} F(\gamma(w)):
\end{aligned}
$$


and we see the need for fractional calculus [16].

As an example of how the technique works we provide in the appendix an explicit non-trivial proof that

$$
\left(\beta^{a}(z) \gamma^{a}(w)\right)\left(\beta^{b}(z) \gamma^{b}(w)\right)=\beta^{a+b}(z) \gamma^{a+b}(w)
$$

Additionally, our explicit verification that our results for the $N$-point functions satisfy the Knizhnik-Zamolodchikov equations may be viewed as a check that fractional calculus does indeed provide us with the requisite properties for Wick contractions as defined in eq.(21) .

\section{The three point function and fusion rules}

Before considering correlators, we want to define our notations and choices as far as dual states are concerned [20, 5]. We use the following mode expansions and definitions:

$$
\begin{aligned}
\beta(z)=\sum_{n \in \mathbb{Z}} \beta_{n} z^{-n-1} & , \quad \gamma(z)=\sum_{n \in \mathbb{Z}} \gamma_{n} z^{-n} \\
: \beta_{n} \gamma_{m}: & =\left\{\begin{array}{l}
\beta_{n} \gamma_{m}, n<0 \\
\gamma_{m} \beta_{n}, n \geq 0
\end{array}\right. \\
j(z) & =-: \gamma(z) \beta(z):=+\partial \phi(z) \\
\phi(z) \phi\left(z^{\prime}\right) & =-\log \left(z-z^{\prime}\right) \\
\varphi(z) \varphi\left(z^{\prime}\right) & =+\log \left(z-z^{\prime}\right) \\
\phi(z) & =q_{\phi}+a_{\phi} \log z+\sum_{n \neq 0} \frac{j_{n}}{-n} z^{-n} \\
\varphi(z) & =q_{\varphi}+a_{\varphi} \log z+\sum_{n \neq 0} \frac{a_{n}}{-n} z^{-n} \\
j_{0} & \equiv a_{\phi} \\
{\left[a_{\phi}, q_{\phi}\right] } & =-1 \\
{\left[a_{\varphi}, q_{\varphi}\right] } & =+1 \\
j(z) & =\sum_{n \in \mathbb{Z}} j_{n} z^{-n-1} \\
j_{n}^{\dagger}=-j_{-n} & , \quad a_{n}^{\dagger}=a_{-n} \\
\gamma_{n}^{\dagger}=\gamma_{-n} & , \quad \beta_{n}^{\dagger}=-\beta_{-n}
\end{aligned}
$$

The ket-vacuum, invariant under both projective $s l_{2}$ and affine zero-mode $s l_{2}$, is $|0\rangle$ satisfying

$$
\begin{aligned}
& \beta_{n}|0\rangle=0=\gamma_{n}|0\rangle=a_{n}|0\rangle=j_{n}|0\rangle, n>0 \\
& \beta_{0}|0\rangle=0=a_{\varphi}|0\rangle=a_{\phi}|0\rangle \\
& \gamma_{0}|0\rangle \neq 0 \\
& L_{n}|0\rangle=0, \quad n \geq-1
\end{aligned}
$$


with

$$
L_{n}=\sum_{m \in \mathbb{Z}}\left(-m: \beta_{n-m} \gamma_{m}:+\frac{1}{2}: a_{n-m} a_{m}:\right)-(n+1) \sqrt{\frac{1}{2 t}} a_{n}
$$

Correspondingly the $s l_{2}$ invariant bra-vacuum, $\left\langle s l_{2}\right|$, satisfies

$$
\begin{aligned}
& \left\langle s l_{2}\right| L_{n}=0, n \leq 1 \\
& \left\langle s l_{2}\right| \beta_{n}=0, n \leq 0 \\
& \left\langle s l_{2}\right| \gamma_{n}=0, n \leq-1 \\
& \left\langle s l_{2}\right| j_{0}=\left\langle s l_{2}\right| a_{\phi}=\left\langle s l_{2}\right| \\
& \left\langle s l_{2}\right| a_{\varphi}=\sqrt{\frac{2}{t}}\left\langle s l_{2}\right| \\
& \left\langle s l_{2} \mid 0\right\rangle=0
\end{aligned}
$$

The last equality is due to the fact that the bra-vacuum defined above carries different charges comparing to the ket-vacuum. In what follows we shall define another bra-vacuum with all the charges at infinity screened. This will be the dual vacuum we are mostly going to use in calculating the correlators.

We define the dual vacuum state in the WZNW free field realization, $\langle 0|$, as

$$
\langle 0|=\left\langle s l_{2}\right| e^{-q_{\phi}} e^{\sqrt{2 / t} q_{\varphi}}
$$

It satisfies:

$$
\begin{aligned}
\langle 0 \mid 0\rangle & =1 \\
\langle 0| \gamma_{0} & =0 \\
\langle 0| \beta_{0} & \neq 0 \\
\langle 0| a_{\varphi} & =0 \\
\langle 0| j_{0}=\langle 0| a_{\phi} & =0 \\
\left\langle 0\left|\beta(z) \gamma\left(z^{\prime}\right)\right| 0\right\rangle & =\frac{1}{z-z^{\prime}}
\end{aligned}
$$

From the dual vacuum we construct dual bra-states of lowest $S L(2)$ weight

$$
\langle j|=\langle 0| e^{j \sqrt{2 / t} q_{\varphi}}
$$

This state indeed satisfies the conditions for being a lowest weight state of the affine algebra:

$$
\begin{aligned}
\langle j| J_{0}^{3} & =j\langle j| \\
\langle j| J_{n}^{-} & =0, n \leq 0 \\
\langle j| J_{n}^{3} & =0, n<0 \\
\langle j| J_{n}^{+} & =0, n<0
\end{aligned}
$$


For the corresponding ket states

$$
|j\rangle=e^{-j \sqrt{2 / t}} q_{\varphi}|0\rangle
$$

we have

$$
\langle j \mid j\rangle=1
$$

and this ket state is similarly a highest weight state of the affine algebra. We notice that

$$
\langle 0| J_{0}^{+}=\langle 0| \beta_{0} \neq 0
$$

Thus we are performing all calculations with an $s l_{2}$ non-invariant bra-vacuum. This gives rise to some complications when we wish to prove projective and global $s l_{2}$ invariance of our correlators. We shall explicitly demonstrate in sect. 7 that the above state, $\langle 0| \beta_{0}$, while not being zero is in fact a BRST exact state in the sense of Felder [21], and that therefore it must be expected to decouple from all correlators. This decoupling we then verify.

Let us now consider the evaluation of the (chiral) three point function

$$
\left\langle j_{3}\left|\phi_{j_{2}}(z, x)\right| j_{1}\right\rangle
$$

where, the dual bra, $\left\langle j_{3}\right|$, and the ket, $\left|j_{1}\right\rangle$, are defined above. Using the free field realizations of $\phi_{j_{2}}(z, x)$, eq.(10), the three point function may be evaluated only provided the "momenta" may be screened away in the standard way [22], and correspondingly $\phi_{j_{2}}(z, x)$ is replaced by the intertwining field, $\left(\phi_{j_{2}}(z, x)\right)_{j_{1}}^{j_{3}}$, which maps a $j_{1}$ highest weight module into a $j_{3}$ highest weight module. Following Felder [21, 2], but using the two screening charges of [15 in eq.(19) instead, we are led to consider the intertwining field

$$
\begin{aligned}
\left(\phi_{j_{2}}(z, x)\right)_{j_{1}}^{j_{3}} & =\oint \prod_{j=1}^{s} \frac{d v_{j}}{2 \pi i} \prod_{i=1}^{r} \frac{d u_{i}}{2 \pi i} \phi_{j_{2}}(z, x) P\left(u_{1}, \ldots, u_{r} ; v_{1}, \ldots, v_{s}\right) \\
P\left(u_{1}, \ldots, u_{r} ; v_{1}, \ldots, v_{s}\right) & =\prod_{j=1}^{s} \beta^{-t}\left(v_{j}\right) e^{-\sqrt{2 t} \varphi\left(v_{j}\right)} \prod_{i=1}^{r} \beta\left(u_{i}\right) e^{\sqrt{2 / t} \varphi\left(u_{i}\right)}
\end{aligned}
$$

This requires that

$$
j_{1}+j_{2}-j_{3}=r-s t
$$

with $r$ and $s$ non negative integers. It is trivial using well known techniques to perform the $\varphi$ part of the Wick contractions. Hence we concentrate on explaining how to perform the $\beta \gamma$ part. First we have to determine the asymptotic expansion in $\gamma$ within $\phi_{j_{2}}(z, x)$. By projective invariance of the three point function, $x$ could be fractionally powered, when $s$ in eq.(36) is nonzero:

$$
\left\langle j_{3}\left|\left(\phi_{j_{2}}(z, x)\right)_{j_{1}}^{j_{3}}\right| j_{1}\right\rangle \propto x^{r-s t}
$$

Hence we should expand asymptotically,

$$
\begin{aligned}
\phi_{j_{2}}(z, x) & =(1+\gamma(z) x)_{(-s t)}^{2 j_{2}} e^{-j_{2} \sqrt{2 / t} \varphi(z)} \\
& =\sum_{n \in \mathbb{Z}}\left(\begin{array}{c}
2 j_{2} \\
n-s t
\end{array}\right)(\gamma(z) x)^{n-s t} e^{-j_{2} \sqrt{2 / t} \varphi(z)}
\end{aligned}
$$


and

$$
\begin{aligned}
& \beta(w)^{-t}(1+\gamma(z) x)_{(-s t)}^{2 j_{2}} \\
= & :\left(\beta(w)+\frac{1}{w-z} \partial_{\gamma(z)}\right)^{-t} \sum_{m \in \mathbb{Z}}\left(\begin{array}{c}
2 j_{2} \\
m-s t
\end{array}\right)(\gamma(z) x)^{m-s t}: \\
= & \sum_{n=0}^{\infty}\left(\begin{array}{c}
-t \\
n
\end{array}\right): \beta^{n}(w)(w-z)^{t+n} \partial_{\gamma(z)}^{-t-n} \sum_{m \in \mathbb{Z}}\left(\begin{array}{c}
2 j_{2} \\
m-s t
\end{array}\right)(\gamma(z) x)^{m-s t}: \\
= & \sum_{n=0}^{\infty}\left(\begin{array}{c}
-t \\
n
\end{array}\right): \beta^{n}(w)(w-z)^{t+n} \frac{\Gamma\left(2 j_{2}+1\right)}{\Gamma\left(2 j_{2}+t+n+1\right)}(1+\gamma(z) x)_{((1-s) t)}^{2 j_{2}+t+n}: x^{-t-n}
\end{aligned}
$$

Similarly, we deduce

$$
\begin{aligned}
& \prod_{i=1}^{s} \beta\left(w_{i}\right)^{-t}(1+\gamma(z) x)_{(-s t)}^{2 j_{2}} \\
= & : \prod_{i=1}^{s}\left(\beta\left(w_{i}\right)+\frac{1}{w_{i}-z} \partial_{\gamma(z)}\right)^{-t} \sum_{m \in \mathbb{Z}}\left(\begin{array}{c}
2 j_{2} \\
m-s t
\end{array}\right)(\gamma(z) x)^{m-s t}: \\
= & : \prod_{i=1}^{s} \sum_{n_{i}=0}^{\infty}\left(\begin{array}{c}
-t \\
n_{i}
\end{array}\right) \beta^{n_{i}}\left(w_{i}\right)\left(w_{i}-z\right)^{t+n_{i}} \partial_{\gamma(z)}^{-t-n_{i}} \sum_{m \in \mathbb{Z}}\left(\begin{array}{c}
2 j_{2} \\
m-s t
\end{array}\right)(\gamma(z) x)^{m-s t}: \\
= & : \prod_{i=1}^{s} \sum_{n_{i}=0}^{\infty}\left(\begin{array}{c}
-t \\
n_{i}
\end{array}\right) \beta^{n_{i}}\left(w_{i}\right)\left(w_{i}-z\right)^{t+n_{i}} \frac{\Gamma\left(2 j_{2}+1\right)}{\Gamma\left(2 j_{2}+s t+\sum_{i} n_{i}+1\right)} \\
\cdot & (1+\gamma(z) x)^{2 j_{2}+s t+\sum_{i} n_{i}}: x^{-s t-\sum_{i} n_{i}}
\end{aligned}
$$

Notice that in these equations $\beta$ and $\gamma$ appear within normal ordering signs with integral powers.

Eqs.(39) and (40) suggest that we consider some kind of generating function, which looks like the exponential function,

$$
F(u)=\sum_{n \in \mathbb{Z}} \frac{1}{\Gamma(n-a+1)}(1+\gamma(z) x)_{(\alpha)}^{n-a} u^{-n+a}
$$

We shall find it useful to use the following rather trivial identity:

$$
(1+\gamma(z) x)^{2 j}=\Gamma(2 j+1) \oint_{0} \frac{d u}{2 \pi i} \frac{1}{u}\left(u^{-1} D\right)^{-2 j} \exp [(1+\gamma(z) x) / u]
$$

where $D$ converts the exponential function into the derivative of that function, in particular it acts on and only on the entire argument of that function. We now prove the following

\section{Lemma}

$$
\beta^{a}(w) \exp [(1+\gamma(z) x) / u]=:\left(\beta(w)+\frac{x / u}{w-z}\right)^{a} D^{a} \exp [(1+\gamma(z) x) / u]:
$$


Proof of lemma:

$$
\begin{aligned}
& \beta^{a}(w) \exp [(1+\gamma(z) x) / u] \\
= & \sum_{k, m, n \in \mathbb{Z}}\left(\begin{array}{c}
a \\
m
\end{array}\right): \beta^{m}(w)(w-z)^{m-a} \partial_{\gamma(z)}^{a-m} \frac{1}{\Gamma(n+1)}\left(\begin{array}{c}
n \\
k
\end{array}\right)(\gamma(z) x)^{n-k}: u^{-n} \\
= & \sum_{k, m, n \in \mathbb{Z}}\left(\begin{array}{c}
a \\
m
\end{array}\right): \beta^{m}(w)(w-z)^{m-a} \gamma^{n-k-a+m}(z): \\
\cdot & \frac{1}{\Gamma(k+1)} \frac{1}{\Gamma(n-k-a+m+1)} x^{n-k-a+m} x^{a-m} u^{-n} \\
= & \sum_{k, m, N \in \mathbb{Z}}\left(\begin{array}{c}
a \\
m
\end{array}\right): \beta^{m}(w)(w-z)^{m-a} x^{a-m} u^{m-a} \gamma^{-a+N-k}(z): \\
\cdot & \left(\begin{array}{c}
-a+N \\
k
\end{array}\right. \\
= & :\left(\beta(w)+\frac{1}{w(u}\right)^{a} D^{a} \exp [(1+\gamma(z) x / u)]:
\end{aligned}
$$

Q.E.D.

We may now calculate the $\beta \gamma$ parts of the contractions in the 3-point function:

$$
\begin{aligned}
& \prod_{i=1}^{r} \beta\left(u_{i}\right) \prod_{j=1}^{s} \beta^{-t}\left(v_{j}\right)(1+\gamma(z) x)^{2 j} \\
= & \oint_{0} \frac{d u}{2 \pi i} \frac{u^{2 j}}{u}: \prod_{i=1}^{r}\left[\beta\left(u_{i}\right)+\frac{x / u}{u_{i}-z}\right] \prod_{j=1}^{s}\left[\beta\left(v_{j}\right)+\frac{x / u}{v_{j}-z}\right]^{-t} \\
\cdot & D^{-2 j+r-s t} \exp \left\{\frac{1+\gamma(z) x}{u}\right\}: \Gamma(2 j+1)
\end{aligned}
$$

When inserted between $\left\langle j_{3}\right|$ and $\left|j_{1}\right\rangle$ to produce $W_{3}^{\beta \gamma}$, the $\beta \gamma$ part of the three point function, we effectively put $\beta \equiv 0 \equiv \gamma$ whereupon the $u$-integration becomes trivial, and we find the result

$$
W_{3}^{\beta \gamma}=\frac{\Gamma\left(2 j_{2}+1\right)}{\Gamma\left(2 j_{2}-r+s t+1\right)} x^{r-s t} \oint \prod_{i=1}^{r} \frac{d u_{i}}{2 \pi i}\left(u_{i}-z\right)^{-1} \prod_{j=1}^{s} \frac{d v_{j}}{2 \pi i}\left(v_{j}-z\right)^{t}
$$

Here we mean to employ the contours discussed by Felder [21]. The $\varphi$ part of the 3-point function is standard. We may put $z=1=x$ using global projective and global $S L(2)$ invariance. We only give the result for admissible representations:

$$
\begin{aligned}
t & =p / q \\
2 j_{i}+1 & =r_{i}-s_{i} t \\
1 & \leq r_{i} \leq p-1 \\
0 & \leq s_{i} \leq q-1
\end{aligned}
$$


where $(p, q)=1$, and $p, q \in \mathcal{N}$.

$$
\begin{aligned}
W_{3}= & \frac{\Gamma\left(2 j_{2}+1\right)}{\Gamma\left(2 j_{2}-r+s t+1\right)} \\
& \oint \prod_{i=1}^{r} \frac{d u_{i}}{2 \pi i} \prod_{j=1}^{s} \frac{d v_{j}}{2 \pi i} \prod_{i_{1}<i_{2}}\left(u_{i_{1}}-u_{i_{2}}\right)^{2 / t} \prod_{j_{1}<j_{2}}\left(v_{j_{1}}-v_{j_{2}}\right)^{2 t} \prod_{i, j}\left(u_{i}-v_{j}\right)^{-2} \\
& \cdot \prod_{i=1}^{r} u_{i}^{\left(1-r_{1}\right) / t+s_{1}}\left(1-u_{i}\right)^{\left(1-r_{2}\right) / t+s_{2}-1} \prod_{j=1}^{s} v_{j}^{r_{1}-1-s_{1} t}\left(1-v_{j}\right)^{r_{2}-1-\left(s_{2}-1\right) t}
\end{aligned}
$$

This integral is exactly of the Dotsenko-Fateev form [22] and, using the Felder contours, may be written down as [21]

$$
\begin{aligned}
W_{3}= & \frac{\Gamma\left(2 j_{2}+1\right)}{\Gamma\left(j_{2}+j_{3}-j_{1}+1\right)} e^{i \pi r\left(r+1-2 r_{1}\right) / t} e^{i \pi t s\left(s-1-2 s_{1}\right)} t^{r s} \\
\cdot & \prod_{j=1}^{r} \frac{\left(1-e^{2 \pi i\left(r_{1}-j\right) / t}\right)\left(1-e^{2 \pi i j / t}\right)}{1-e^{2 \pi i / t}} \prod_{j=1}^{s} \frac{\left(1-e^{2 \pi i t\left(s_{1}+1-j\right)}\right)\left(1-e^{2 \pi i t j}\right)}{1-e^{2 \pi i t}} \\
\cdot & \prod_{i=1}^{r} \frac{\Gamma(i / t)}{\Gamma(1 / t)} \prod_{i=1}^{s} \frac{\Gamma(i t-s)}{\Gamma(t)} \\
\cdot & \prod_{i=0}^{r-1} \frac{\Gamma\left(s_{1}+1+\left(1-r_{1}+i\right) / t\right) \Gamma\left(s_{2}+\left(1-r_{2}+i\right) / t\right)}{\Gamma\left(s_{1}+s_{2}+1-2 s+\left(r-r_{1}-r_{2}+i+1\right) / t\right)} \\
& \cdot \prod_{i=0}^{s-1} \frac{\Gamma\left(r_{1}-r+\left(i-s_{1}\right) t\right) \Gamma\left(r_{2}-r+\left(1-s_{2}+i\right) t\right)}{\Gamma\left(r_{1}-r+r_{2}+\left(s-s_{1}-s_{2}+i\right) t\right)}
\end{aligned}
$$

The analysis of this expression in terms of fusion rules is standard [21]. The result may be written as follows:

$$
\begin{aligned}
1+\left|r_{1}-r_{2}\right| & \leq r_{3} \leq p-1-\left|r_{1}+r_{2}-p\right| \\
\left|s_{1}-s_{2}\right| & \leq s_{3} \leq q-1-\left|s_{1}+s_{2}-q+1\right|
\end{aligned}
$$

The first line of these fusion rules is well known for the case, $q=1$, of integrable representations, and it was obtained in the general case in [2]. The second was obtained by Awata and Yamada [23] by considering the conditions for decoupling of null-states, and by Feigin and Malikov [24] by cohomological methods. In addition these authors provide a fusion rule ((II) for [23], (I) for [24]), which we do not get in the free field realization. We do not know if there exist conformal field theories with non vanishing couplings respecting those.

We conclude this section by making a comment on the possible $S L(2)$ representations carried by the intertwining field $\left(\phi_{j_{2}}(z, x)\right)_{j_{1}}^{j_{3}}$. Clearly, on the ket-vacuum,

$$
\begin{aligned}
\left(\phi_{j_{2}}(z, x)\right)_{j_{1}}^{j_{3}}|0\rangle & =\left(\phi_{j_{2}}(z, x)\right)_{0}^{j_{2}}|0\rangle \delta_{j_{1}, 0} \\
& =e^{x J_{0}^{-}} e^{z L_{-1}}\left|j_{2}\right\rangle \delta_{j_{1}, 0}
\end{aligned}
$$

is in a highest weight representation of the $S L(2)$ current algebra with the highest weight state $\left|j_{2}\right\rangle$. On the other hand, as it will be explicitly checked in sect. 4, on the dual 
vacuum state,

$$
\begin{aligned}
\langle 0|\left(\phi_{j_{2}}(z, x)\right)_{j_{1}}^{j_{3}} z^{\frac{2 j_{2}\left(j_{2}+1\right)}{t}} x^{-2 j_{2}} & =\langle 0|\left(\phi_{j_{2}}(z, x)\right)_{j_{2}}^{0} z^{\frac{2 j_{2}\left(j_{2}+1\right)}{t}} x^{-2 j_{2}} \\
& =\left\langle j_{2}\right| e^{L_{1} / z} e^{-J_{0}^{+} / x}
\end{aligned}
$$

is in a lowest weight representation of the $S L(2)$ current algebra with the lowest weight state $\left\langle j_{2}\right|$. When sandwiched in the middle of the correlator, however, the intertwining field $\left(\phi_{j_{2}}(z, x)\right)_{j_{1}}^{j_{3}}$ could carry representations belonging to the continuous series of the $S L(2)$ algebra, in which neither a highest weight nor a lowest weight state exists, when both $j_{1}+j_{2}-j_{3}$ and $j_{1}-j_{2}-j_{3}$ are non-integers. This means we decompose $\phi_{j_{2}}(z, x)_{j_{1}}^{j_{3}}$ into $J_{0}^{3}$ eigenstates,

$$
\begin{aligned}
{\left[J_{0}^{3},\left(\phi_{j_{2}, \lambda}(z, x)\right)_{j_{1}}^{j_{3}}\right] } & =\lambda\left(\phi_{j_{2}, \lambda}(z, x)\right)_{j_{1}}^{j_{3}} \\
\lambda & \neq \pm j_{2}(\bmod ) 1
\end{aligned}
$$

Although in that case $\left(\phi_{j_{2}}(z, x)\right)_{j_{1}}^{j_{3}}$ does not correspond to a highest weight representation, it maps a $j_{1}$ highest weight representation to a $j_{3}$ highest weight representation.

\section{The most general $N$-point function for degenerate representations on the sphere}

We wish to evaluate the conformal block

$$
W_{N}=\left\langle j_{N}\left|\left[\phi_{j_{N-1}}\left(z_{N-1}, x_{N-1}\right)\right]_{\iota_{N-2}}^{j_{N}} \ldots\left[\phi_{j_{n}}\left(z_{n}, x_{n}\right)\right]_{\iota_{n-1}}^{\iota_{n}} \ldots\left[\phi_{j_{2}}\left(z_{2}, x_{2}\right)\right]_{j_{1}}^{\iota_{2}}\right| j_{1}\right\rangle
$$

Thus we have primary fields (chiral vertex operators) at points

$$
z_{1}=0, z_{2}, \ldots, z_{N-1}, z_{N}=\infty
$$

having $x$ values

$$
x_{1}=0, x_{2}, \ldots, x_{N-1}, x_{N}=\infty
$$

We parametrize

$$
\begin{aligned}
j_{1}+j_{2}-\iota_{2} & =\rho_{2}-\sigma_{2} t \\
\iota_{2}+j_{3}-\iota_{3} & =\rho_{3}-\sigma_{3} t \\
& \vdots \\
\iota_{n-1}+j_{n}-\iota_{n} & =\rho_{n}-\sigma_{n} t \\
& \vdots \\
\iota_{N-2}+j_{N-1}-j_{N} & =\rho_{N-1}-\sigma_{N-1} t \\
2 j_{i}+1 & =r_{i}-s_{i} t
\end{aligned}
$$

with $\sigma_{n}, \rho_{n}$ non negative integers. We then get for the $\beta \gamma$ part of the correlator, denoting by $w(n, i)$ and $v(n, k)$ the positions of the $i$ 'th and the $k$ 'th screening currents of the first 
and second kinds respectively in eq. (19) around the n'th primary field:

$$
\begin{aligned}
W_{N}^{\beta \gamma}= & \left\langle j_{N}\left|\left(1+x_{n} \gamma\left(z_{n}\right)\right)^{2 j_{n}} \prod_{k=1}^{\sigma_{n}}[\beta(v(n, k))]^{-t} \prod_{n=2}^{N-1} \prod_{i=1}^{\rho_{n}} \beta(w(n, i))\right| j_{1}\right\rangle \\
= & \oint_{0} \prod_{n=2}^{N-1} \frac{d u_{n}}{2 \pi i} \prod_{i=1}^{\rho_{n}} \prod_{k=1}^{\sigma_{n}} \\
& \left\langle j_{N}\right|:\left(\beta(w(n, i))+\frac{x_{n} / u_{n}}{w(n, i)-z_{n}}\right)\left(\beta(v(n, k))+\frac{x_{n} / u_{n}}{v(n, k)-z_{n}}\right)^{-t} \\
\cdot & u_{n}^{2 j_{n}} D_{n}^{-2 j_{n}+\rho_{n}-\sigma_{n} t} \exp \left\{\frac{1+x_{n} \gamma\left(z_{n}\right)}{u_{n}}\right\} \Gamma\left(2 j_{n}+1\right) \frac{1}{u_{n}}:\left|j_{1}\right\rangle \\
= & \oint_{0} \prod_{n=2}^{N-1} \frac{d u_{n}}{2 \pi i} \prod_{i=1}^{\rho_{n}}\left(\sum_{\ell=2}^{N-1} \frac{x_{\ell} / u_{\ell}}{w(n, i)-z_{\ell}}\right) \prod_{k=1}^{\sigma_{n}}\left(\sum_{\ell=2}^{N-1} \frac{x_{\ell} / u_{\ell}}{v(n, k)-z_{\ell}}\right)^{-t} \\
\cdot & u_{n}^{2 j_{n}-1} D_{n}^{-2 j_{n}+\rho_{n}-\sigma_{n} t} \exp \left\{\frac{1}{u_{n}}\right\} \Gamma\left(2 j_{n}+1\right)
\end{aligned}
$$

where we have used the techniques already developed for the three point function. In particular, in the second equality we applied the lemma of the previous section, and in the last equality we kept doing that until normal ordering signs surround all operators, at which point the calculation is completed by putting $\beta$ 's and $\gamma$ 's under normal ordering signs equal to zero. Conforming with the discussion in the previous section we may also throw away all derivatives on the exponential (they are with respect to the full argument of the exponential), but their presence serve to remind us in some cases, what representations would conveniently be used. In the sequel we drop these derivatives. It is straightforward to write down the contribution from the $\varphi$ part of the free field realization. It is

$$
\begin{aligned}
W_{N}^{\varphi}= & \prod_{1 \leq m<n \leq N-1}\left(z_{m}-z_{n}\right)^{2 j_{m} j_{n} / t} \prod_{n=2}^{N-1} \prod_{i=1}^{\rho_{n}} \prod_{m=1}^{N-1}\left(w(n, i)-z_{m}\right)^{-2 j_{m} / t} \\
& \cdot \prod_{n=2}^{N-1} \prod_{k=1}^{\sigma_{n}} \prod_{m=1}^{N-1}\left(v(n, k)-z_{m}\right)^{2 j_{m}} \prod_{(n, i)<\left(n^{\prime}, i^{\prime}\right)}\left(w(n, i)-w\left(n^{\prime}, i^{\prime}\right)\right)^{2 / t} \\
& \cdot \prod_{(n, k)<\left(n^{\prime}, k^{\prime}\right)}\left(v(n, k)-v\left(n^{\prime}, k^{\prime}\right)\right)^{2 t} \prod_{(n, i),\left(n^{\prime}, k\right)}\left(w(n, i)-v\left(n^{\prime}, k\right)\right)^{-2}
\end{aligned}
$$

Here we have introduced a rather arbitrary ordering of indices, for example as

$$
(n, i)<\left(n^{\prime}, i^{\prime}\right)
$$

if either $n<n^{\prime}$ or $n=n^{\prime}, \quad i<i^{\prime}$.

Let us summarize our findings in a more compact notation: Let

$$
\begin{aligned}
& M=\sum_{m=2}^{N-1}\left(\rho_{m}+\sigma_{m}\right) \\
& w_{i} \quad i=1, \ldots, M
\end{aligned}
$$


collectively denote the position of all screening charges:

$$
\left\{w_{i}\right\}=\{w(n, i), v(n, k)\}
$$

Further, let

$$
\begin{aligned}
k_{i} & =\left\{\begin{array}{rl}
-1 & i=1, \ldots, \sum_{m} \rho_{m} \\
t & i=\sum_{m} \rho_{m}+1, \ldots, M
\end{array}\right. \\
B\left(w_{i}\right) & \equiv \sum_{\ell=1}^{N-1} \frac{x_{\ell} / u_{\ell}}{w_{i}-z_{\ell}}
\end{aligned}
$$

(here $x_{1}=0$ ). Then the integrand of the $N$-point function is given by (we use the same letters for the integrated expressions, we hope this will not cause confusion)

$$
W_{N}=W_{B} W_{N}^{\varphi} F
$$

with

$$
\begin{aligned}
W_{N}^{\beta \gamma} & =W_{B} F \\
W_{B} & =\prod_{i=1}^{M} B\left(w_{i}\right)^{-k_{i}} \\
W_{N}^{\varphi} & =\prod_{m<n}\left(z_{m}-z_{n}\right)^{2 j_{m} j_{n} / t} \prod_{i=1}^{M} \prod_{m=1}^{N-1}\left(w_{i}-z_{m}\right)^{2 k_{i} j_{m} / t} \prod_{i<j<M}\left(w_{i}-w_{j}\right)^{2 k_{i} k_{j} / t} \\
F & =\prod_{m=2}^{N-1} \Gamma\left(2 j_{m}+1\right) u_{m}^{2 j_{m}-1} e^{\frac{1}{u_{m}}}
\end{aligned}
$$

This integrand is to be integrated over the $u_{m}$ 's along contours enclosing $u_{m}=0$, and over the $w_{i}$ 's along the Felder contours.

We believe the above general closed expression for integral representation of the $N$ point function to be useful for further development, in particular integrations over the auxiliary variables, $u_{\ell}, \ell=2, \ldots, N-1$ seem tractable as they stand. If for some reason, one needs to get rid of these integrations, it is not too difficult. As an example, we provide an explicit form for the result for integrable representations. First we define the following notation:

Let

$$
\begin{aligned}
J_{N} & =\{2,3, \ldots, N-1\} \\
I_{N} & =\left\{(n, i) \mid n=2, \ldots, N-1, i=1,2, \ldots, \rho_{n}\right\} \\
\mathcal{F}_{N} & =\left\{\text { maps, } f, \text { from } I_{N} \text { to } J_{N}\right\}
\end{aligned}
$$

For $t=k+2$ integer, all $\sigma^{\prime}$ s are $=0$. In this case we may then write

$$
W_{N}^{\beta \gamma}=\oint_{0} \prod_{n=2}^{N-1} \frac{d u_{n}}{2 \pi i} u_{n}^{2 j_{n}} \frac{e^{1 / u_{n}}}{u_{n}}\left(2 j_{n}\right) ! \prod_{(n, i) \in I_{N}}\left(\sum_{\ell=2}^{N-1} \frac{x_{\ell} / u_{\ell}}{w(n, i)-z_{\ell}}\right)
$$




$$
\begin{aligned}
= & \oint_{0} \prod_{n=2}^{N-1} \frac{d u_{n}}{2 \pi i} u_{n}^{2 j_{n}} \frac{e^{1 / u_{n}}}{u_{n}}\left(2 j_{n}\right) ! \sum_{f \in \mathcal{F}_{N}} \prod_{(n, i) \in I_{N}}\left(\frac{x_{f(n, i)} / u_{f(n, i)}}{w(n, i)-z_{f(n, i)}}\right) \\
= & \oint_{0} \prod_{n=2}^{N-1} \frac{d u_{n}}{2 \pi i} u_{n}^{2 j_{n}} \frac{e^{1 / u_{n}}}{u_{n}}\left(2 j_{n}\right) ! \\
& \sum_{f \in \mathcal{F}_{N}} \prod_{\ell \in J_{N}}\left(x_{\ell} / u_{\ell}\right)^{\left|f^{-1}(\ell)\right|} \prod_{(n, i) \in f^{-1}(\ell)}\left(w(n, i)-z_{\ell}\right)^{-1} \\
= & \sum_{f \in \mathcal{F}_{N}} \prod_{\ell \in J_{N}} x_{\ell}^{\left|f^{-1}(\ell)\right|} \frac{\left(2 j_{\ell}\right) !}{\left(2 j_{\ell}-\left|f^{-1}(\ell)\right|\right) !} \prod_{(n, i) \in f^{-1}(\ell)}\left(w(n, i)-z_{\ell}\right)^{-1}
\end{aligned}
$$

A similar but even more complicated sum formula obtains in the general case.

We now have two ways of calculating the conformal block corresponding to $N$ primaries. The way so far described is by using (part of) projective invariance (to be discussed further later) and global $s l_{2}$ invariance to work it out as

$$
\begin{aligned}
& W_{N}^{(I)}\left(z_{N}=\infty, x_{N}=\infty, z_{N-1}, x_{N-1}, \ldots, z_{2}, x_{2}, z_{1}=0, x_{1}=0\right) \\
= & \left\langle j_{N}\left|\left[\phi_{j_{N-1}}\left(z_{N-1}, x_{N-1}\right)\right]_{\iota_{N-2}}^{j_{N} \ldots}\left[\phi_{j_{2}}\left(z_{2}, x_{2}\right)\right]_{j_{1}}^{\iota_{2}}\right| j_{1}\right\rangle
\end{aligned}
$$

However, obviously, we may also use our technique to evaluate the same $N$-point conformal block as

$$
\begin{aligned}
& W_{N}^{(I I)}\left(z_{N}, x_{N}, z_{N-1}, x_{N-1}, \ldots, z_{2}, x_{2}, z_{1}, x_{1}\right) \\
= & \langle 0|\left[\phi_{j_{N}}\left(z_{N}, x_{N}\right)\right]_{j_{N}}^{0}\left[\phi_{j_{N-1}}\left(z_{N-1}, x_{N-1}\right)\right]_{\iota_{N-2}}^{j_{N}} \ldots \\
& \ldots\left[\phi_{j_{2}}\left(z_{2}, x_{2}\right)\right]_{j_{1}}^{l_{2}}\left[\phi_{j_{1}}\left(z_{1}, x_{1}\right)\right]_{0}^{j_{1}}|0\rangle
\end{aligned}
$$

We now want to demonstrate that up to normalization these expressions are equivalent in the appropriate limits. This procedure has also been mentioned in section 3 when discussing different representations mapped into one another by the intertwining field. Here is the direct check. Notice that the second form involves more screening charges around the last field than the first one. We shall see that these extra screenings give rise to a constant contribution in the limit $z_{N}, x_{N} \rightarrow \infty$. But for finite $z_{N}$ and $x_{N}$, unlike in the case of the conformal minimal models, there does not seem to be any simple way of getting a conjugate field, which would get rid of the extra screening charges.

It is clear that in the limit $z_{1}, x_{1} \rightarrow 0$ the second formulation coincides with the first. In particular, the second formulation involves no extra screening operators. Thus we shall concentrate on the limit $z_{N}, x_{N} \rightarrow \infty$.

As before, we let $w_{i}$ denote the position of screening operators in the first case, $W_{N}^{(I)}$, and we let $i$ run over the same set as in the first case. Further, we let $z_{n}, x_{n}$ denote the arguments as in the first case and $n=1,2, \ldots, N-1$ runs over the same set as in the first case. The new feature in the second case is:

(i) the appearance of

$$
j_{N}+j_{N}-0=r_{N}-1-s_{N} t=\rho_{N}-\sigma_{N} t
$$

extra screening operators, the positions of which we denote by

$$
w_{i_{N}}^{N}, i_{N}=1, \ldots, \rho_{N}+\sigma_{N}
$$




$$
k_{i_{N}}^{N}=\left\{\begin{aligned}
-1 & , \quad i_{N}=1, \ldots, \rho_{N} \\
t & , \quad i_{N}=\rho_{N}+1, \ldots, \rho_{N}+\sigma_{N}
\end{aligned}\right.
$$

and

(ii) an extra $u$-integration over a variable we call $u_{N}$.

Then we want to consider the limit as $z_{N}, x_{N} \rightarrow \infty$ (letting $W_{N}^{(I)}$ stand for the integrand in an appropriate way) of

$$
\begin{aligned}
& z^{\frac{2 j_{N}\left(j_{N}+1\right)}{t}} x^{-2 j_{N}} W_{N}^{(I I)} \\
= & z^{\frac{2 j_{N}\left(j_{N}+1\right)}{t}} x^{-2 j_{N}} \Gamma\left(2 j_{N}+1\right) \prod_{i_{N}} B^{-k_{i_{N}}^{N}}\left(w_{i_{N}}^{N}\right) \prod_{i_{N}<j_{N}}\left(w_{i_{N}}^{N}-w_{j_{N}}^{N}\right)^{2 k_{i_{N}}^{N} k_{j_{N}}^{N} / t} \\
\cdot & \prod_{i, i_{N}}\left(w_{i_{N}}^{N}-w_{i}\right)^{2 k_{i_{N}}^{N} k_{i} / t} \prod_{i_{N}}\left(z_{N}-w_{i_{N}}^{N}\right)^{2 k_{i_{N}}^{N} j_{N} / t} \prod_{i_{N}, n}\left(w_{i_{N}}^{N}-z_{n}\right)^{2 k_{i_{N}}^{N} j_{n} / t} \\
\cdot & \prod_{n}\left(z_{N}-z_{n}\right)^{2 j_{N} j_{n} / t} \prod_{i}\left(z_{N}-w_{i}\right)^{2 j_{N} k_{i} / t} u_{N}^{2 j_{N}-1} e^{1 / u_{N}} \frac{d u_{N}}{2 \pi i} W_{N}^{(I)} \prod_{i_{N}} d w_{i_{N}}^{N}
\end{aligned}
$$

where the function $B(w)$ is defined with one more term than for case (I), cf. eq.(60) . We now use that

$$
\begin{aligned}
-\sum_{i_{N}} k_{i_{N}}^{N} & =\rho_{N}-\sigma_{N} t=2 j_{N} \\
\sum_{i} k_{i} & =-\sum_{n} j_{n}+j_{N}
\end{aligned}
$$

In the limit, $z_{N} \rightarrow \infty, x_{N} \rightarrow \infty$ we find

$$
\begin{aligned}
w_{i_{N}}^{N} / z_{N} & =\tilde{w}_{i_{N}}^{N} \\
w_{i} / z_{N} & \rightarrow 0 \\
z_{n} / z_{N} & \rightarrow 0
\end{aligned}
$$

with $\tilde{w}_{i_{N}}^{N}$ finite. Also

Hence

$$
B\left(w_{i_{N}}^{N}\right) \sim \frac{x_{N} / u_{N}}{\left(1-\tilde{w}_{i_{N}}^{N}\right) z_{N}}
$$

$$
\begin{aligned}
& \lim _{z_{N}, x_{N} \rightarrow \infty} z^{\frac{2 j_{N}\left(j_{N}+1\right)}{t}} x^{-2 j_{N}} W_{N}^{(I I)} \\
& \sim z^{2 \frac{j_{N}\left(j_{N}+1\right)}{t}} x_{N}^{-2 j_{N}} x_{N}^{-\sum_{i_{N}} k_{i_{N}}^{N}} u_{N}^{\sum_{i_{N}} k_{i_{N}}^{N}} z_{N}^{\sum_{i_{N}} k_{i_{N}}^{N}} \prod_{i_{N}}\left(1-\tilde{w}_{i_{N}}^{N}\right)^{k_{i_{N}}^{N}} \\
& \cdot \prod_{i_{N}<j_{N}}\left(\tilde{w}_{i_{N}}^{N}-\tilde{w}_{j_{N}}^{N}\right)^{2 k_{i_{N}}^{N} k_{j_{N}}^{N} / t} z_{N}^{\sum_{i_{N}<j_{N}} 2 k_{i_{N}}^{N} k_{j_{N}}^{N} / t} \\
& \cdot \prod_{i_{N}, i}\left(\tilde{w}_{i_{N}}^{N}\right)^{2 k_{i_{N}}^{N} k_{i} / t} z_{N}^{\sum_{i_{N}, i} 2 k_{i_{N}}^{N} k_{i} / t} \prod_{i_{N}}\left(1-\tilde{w}_{i_{N}}^{N}\right)^{2 k_{i_{N}}^{N} j_{N} / t} z_{N}^{\sum_{i_{N}} 2 k_{i_{N}}^{N} j_{N} / t} \\
& \prod_{i_{N}, n}\left(\tilde{w}_{i_{N}}^{N}\right)^{2 k_{i_{N}}^{N} j_{n} / t} z_{N}^{\sum_{i_{N}, n} 2 k_{i_{N}}^{N} j_{n} / t} z_{N}^{\sum_{n} 2 j_{N} j_{n} / t} z_{N}^{\sum_{i} 2 j_{N} k_{i} / t} \\
& u_{N}^{2 j_{N}-1} e^{1 / u_{N}} \Gamma\left(2 j_{N}+1\right) \frac{d u_{N}}{2 \pi i} W_{N}^{(I)} \prod_{i_{N}} d \tilde{w}_{i_{N}}^{N} z_{N}^{\rho_{N}+\sigma_{N}}
\end{aligned}
$$


We may evaluate the total power of $z_{N}$ as

$$
\begin{aligned}
& \frac{2}{t} j_{N}\left(j_{N}+1\right)-2 j_{N}+\frac{1}{t}\left[\left(\sum_{i_{N}} k_{i_{N}}^{N}\right)^{2}-\sum_{i_{N}}\left(k_{i_{N}}^{N}\right)^{2}\right]+\frac{2}{t}\left(-2 j_{N}\right)\left(-\sum_{n} j_{n}+j_{N}\right) \\
+ & \frac{2}{t} j_{N}\left(-2 j_{N}\right)+\frac{2}{t}\left(-2 j_{N}\right) \sum_{n} j_{n}+\frac{2}{t} j_{N} \sum_{n} j_{n}+\frac{2}{t} j_{N}\left(-\sum_{n} j_{n}+j_{N}\right)+\rho_{N}+\sigma_{N} \\
= & 0
\end{aligned}
$$

which merely shows that the intertwining field, $\left[\phi_{j_{N}}\left(z_{N}, x_{N}\right)\right]_{j_{N}}^{0}$ indeed has the right scaling dimension in our formalism. Similarly the total power of zero for $x_{N}$ says that we treat the field in our formalism with correct global $s l_{2}$ properties. The $u_{N}$-integrand becomes trivial, involving only

$$
u_{N}^{-1} e^{1 / u_{N}}
$$

The dependence on $\tilde{w}_{i_{N}}^{N}$ becomes

$$
\begin{aligned}
& \prod_{i_{N}}\left(1-\tilde{w}_{i_{N}}^{N}\right)^{k_{i_{N}}^{N}} \prod_{i_{N}<j_{N}}\left(\tilde{w}_{i_{N}}^{N}-\tilde{w}_{j_{N}}^{N}\right)^{2 k_{i_{N}}^{N} k_{j_{N}}^{N} / t} \\
& \left(\prod_{i_{N}}\left(\tilde{w}_{i_{N}}^{N}\right)^{2 k_{i_{N}}^{N} / t}\right)^{\left(-\sum_{n} j_{n}+j_{N}\right)} \prod_{i_{N}}\left(1-\tilde{w}_{i_{N}}^{N}\right)^{2 k_{i_{N}}^{N} j_{N} / t} \\
& \left(\prod_{i_{N}}\left(\tilde{w}_{i_{N}}^{N}\right)^{2 k_{i_{N}}^{N} / t}\right)^{\sum_{n} j_{n}}
\end{aligned}
$$

So that this yields an integral over the $\tilde{w}_{i_{N}}^{N}$ 's which is independent of the remaining parameters of the correlator, except $j_{N}$, and thus merely contributes to the normalization of the state $\left\langle j_{N}\right|$.

\section{The 4-point function}

Using the general results for the $N$-point correlators, we now specialize by way of illustration to the case of 4-point correlation functions. First consider the $\beta \gamma$ part,

$$
\left\langle j_{4}\left|\left[\phi_{j_{3}}\left(z_{3}, x_{3}\right)\right]_{j}^{j_{4}}\left[\phi_{j_{2}}\left(z_{2}, x_{2}\right)\right]_{j_{1}}^{j}\right| j_{1}\right\rangle_{\beta \gamma}
$$

with the notation

$$
\begin{aligned}
& j_{1}+j_{2}-j=\rho_{2}-\sigma_{2} t \\
& j+j_{3}-j_{4}=\rho_{3}-\sigma_{3} t
\end{aligned}
$$

So as far as the $\beta \gamma$ part is concerned this means we get

$$
\begin{aligned}
& \oint_{0} \frac{d u_{2}}{2 \pi i} \frac{d u_{3}}{2 \pi i} \frac{1}{u_{2} u_{3}} u^{\rho_{2}-\sigma_{2} t} \\
& \cdot \prod_{i_{3}=1}^{\rho_{3}}\left[\frac{x_{2} / u}{w\left(3, i_{3}\right)-z_{2}}+\frac{x_{3}}{w\left(3, i_{3}\right)-z_{3}}\right] \prod_{l_{3}=1}^{\sigma_{3}}\left[\frac{x_{2} / u}{v\left(3, l_{3}\right)-z_{2}}+\frac{x_{3}}{v\left(3, l_{3}\right)-z_{3}}\right]^{-t}
\end{aligned}
$$




$$
\begin{array}{ll}
\cdot & \prod_{i_{2}=1}^{\rho_{2}}\left[\frac{x_{2} / u}{w\left(2, i_{2}\right)-z_{2}}+\frac{x_{3}}{w\left(2, i_{2}\right)-z_{3}}\right] \prod_{l_{2}=1}^{\sigma_{2}}\left[\frac{x_{2} / u}{v\left(2, l_{2}\right)-z_{2}}+\frac{x_{3}}{v\left(2, l_{2}\right)-z_{3}}\right]^{-t} \\
\cdot & \left(u_{3}^{-1} D_{3}\right)^{-2 j_{3}+\rho_{3}-\sigma_{3} t} \exp \left\{\frac{1}{u_{3}}\right\} \Gamma\left(2 j_{3}+1\right) \\
\cdot & \left(u_{2}^{-1} D_{2}\right)^{-2 j_{2}+\rho_{2}-\sigma_{2} t} \exp \left\{\frac{1}{u_{2}}\right\} \Gamma\left(2 j_{2}+1\right)
\end{array}
$$

where, we have let

$$
u \equiv u_{2} / u_{3}
$$

Again the (somewhat misleading) notation is that the $D$ 's are derivatives with respect to the entire argument of the relevant exponentials.

Now we notice the following identity for fractional derivatives of exponentials:

$$
D_{x}^{a} \exp (x) D_{y}^{b} \exp (y)=D^{a+b} \exp (x+y)
$$

We want to consider a change of variables from $\left(u_{2}, u_{3}\right)$ to $\left(u_{2}, u\right)$. The integration measure is

$$
\begin{gathered}
\frac{d u_{2}}{u_{2}} \frac{d u_{3}}{u_{3}}=-\frac{d u_{2}}{u_{2}} \frac{d u}{u} \\
2 j_{2}+2 j_{3}-\rho_{3}+\sigma_{3} t-\rho_{2}+\sigma_{2} t=2 j_{2}+2 j_{3}-\left(j_{3}+j-j_{4}\right)-\left(j_{2}+j_{1}-j\right)=j_{2}+j_{3}+j_{4}-j_{1} \equiv J_{1}
\end{gathered}
$$

We have introduced the notation

$$
J_{i} \equiv j_{1}+j_{2}+j_{3}+j_{4}-2 j_{i}
$$

Using the generalized exponential identity, we obtain the following $u$ and $u_{2}$ dependence:

$$
\frac{d u d u_{2}}{u u_{2}} u^{-2 j_{3}+\rho_{2}-\sigma_{2} t+\rho_{3}-\sigma_{3} t} u_{2}^{J_{1}} D^{-J_{1}} \exp \left\{\frac{1+u}{u_{2}}\right\}
$$

Now the integral over $u_{2}$ will produce the factor

$$
\frac{(1+u)^{J_{1}}}{\Gamma\left(J_{1}+1\right)}
$$

We are left with the following integral:

$$
\begin{aligned}
& \oint_{0} \frac{d u}{2 \pi i} \prod_{i_{3}=1}^{\rho_{3}}\left[\frac{x_{2}}{w\left(3, i_{3}\right)-z_{2}}+\frac{x_{3} u}{w\left(3, i_{3}\right)-z_{3}}\right] \prod_{l_{3}=1}^{\sigma_{3}}\left[\frac{x_{2}}{v\left(3, l_{3}\right)-z_{2}}+\frac{x_{3} u}{v\left(3, l_{3}\right)-z_{3}}\right]^{-t} \\
\cdot & \prod_{i_{2}=1}^{\rho_{2}}\left[\frac{x_{2}}{w\left(2, i_{2}\right)-z_{2}}+\frac{x_{3} u}{w\left(2, i_{2}\right)-z_{3}}\right] \prod_{l_{2}=1}^{\sigma_{2}}\left[\frac{x_{2}}{v\left(2, l_{2}\right)-z_{2}}+\frac{x_{3} u}{v\left(2, l_{2}\right)-z_{3}}\right]^{-t} \\
\cdot & u^{-2 j_{3}-1} \frac{(1+u)^{J_{1}}}{\Gamma\left(J_{1}+1\right)} \Gamma\left(2 j_{3}+1\right) \Gamma\left(2 j_{2}+1\right)
\end{aligned}
$$

To write the final result for the 4-point function in a more compact form, let us collectively denote the positions for both kinds of screening charges as

$$
w_{i}, i=1, \ldots, \rho_{2}+\rho_{3}+\sigma_{2}+\sigma_{3} \equiv M
$$


Then the complete expression for the 4-point function is

$$
\begin{aligned}
& \left\langle j_{4}\left|\left[\phi_{j_{3}}\left(z_{3}, x_{3}\right)\right]_{j}^{j_{4}}\left[\phi_{j_{2}}\left(z_{2}, x_{2}\right)\right]_{j_{1}}^{j}\right| j_{1}\right\rangle \\
= & \prod_{m<n}\left(z_{m}-z_{n}\right)^{2 j_{m} j_{n} / t} \Gamma\left(2 j_{3}+1\right) \Gamma\left(2 j_{2}+1\right) \\
\cdot & \oint_{0} \frac{d u}{2 \pi i} \prod_{i=1}^{M} \oint \frac{d w_{i}}{2 \pi i}\left[\frac{x_{2}}{w_{i}-z_{2}}+\frac{x_{3} u}{w_{i}-z_{3}}\right]^{-k_{i}} \prod_{i<j}\left(w_{i}-w_{j}\right)^{\frac{2 k_{i} k_{j}}{t}} \\
& \prod_{i=1}^{M} \prod_{l=1}^{3}\left(w_{i}-z_{l}\right)^{\frac{2 k_{i} j_{l}}{t}} u^{-2 j_{3}-1} \frac{(1+u)^{J_{1}}}{\Gamma\left(J_{1}+1\right)}
\end{aligned}
$$

where only one auxiliary integration over $u$ is involved. Let us now work out some specific examples. In those example we shall fix $z_{3}=x_{3}=1$. Due to projective invariance in both $z$ and $x$ spaces, the more general case can be easily recovered.

Example 1:

$$
\begin{aligned}
& j_{1}=j_{2}=j_{3}=j_{4}=1 / 2, \quad j=0, \quad J_{1}=1 \\
& \rho_{2}=1, \quad \sigma_{2}=\rho_{3}=\sigma_{3}=0
\end{aligned}
$$

In this case the $u$ integral can be explicitly carried out, leaving the result

$$
\begin{aligned}
& \left\langle 1 / 2\left|\left[\phi_{1 / 2}(1,1)\right]_{0}^{1 / 2}\left[\phi_{1 / 2}(z, x)\right]_{1 / 2}^{0}\right| 1 / 2\right\rangle \\
= & -((1-z) z)^{1 /(2 t)} \oint \frac{d w}{2 \pi i}\left(\frac{1}{1-w}+\frac{x}{z-w}\right)((1-w)(z-w) w)^{-1 / t} \\
= & (-)^{1 / t}((1-z) z)^{1 /(2 t)}\left\{\left(\begin{array}{c}
-1 / t \\
1 / t-1
\end{array}\right) z^{-2 / t+1} F(1 / t+1,-1 / t+1 ;-2 / t+2 ; z)\right. \\
+ & \left.x\left(\begin{array}{c}
-1 / t-1 \\
1 / t-1
\end{array}\right) z^{-2 / t} F(1 / t,-1 / t+1 ;-2 / t+1 ; z)\right\}
\end{aligned}
$$

Here the $w$-integration contour goes through $z$ and encircles $w=0$, and $F(a, b ; c ; z)$ is the hypergeometric function.

Example 2: In this example we shall consider the case where a second screening charge is needed.

$$
\begin{aligned}
& j_{1}=-t / 2, \quad j_{2}=1 / 2, \quad j_{3}=-t / 2, \quad j_{4}=1 / 2, \quad j=1 / 2-t / 2, \quad J_{1}=1 \\
& \sigma_{3}=1, \quad \sigma_{2}=\rho_{2}=\rho_{3}=0
\end{aligned}
$$

In this case both the $u$ and $w$ integrals can be carried out, and yields

$$
\begin{aligned}
& \left\langle 1 / 2\left|\left[\phi_{-t / 2}(1,1)\right]_{1 / 2-t / 2}^{1 / 2}\left[\phi_{1 / 2}(z, x)\right]_{-t / 2}^{1 / 2-t / 2}\right|-t / 2\right\rangle \\
= & (z(1-z))^{-1 / 2} \oint \frac{d w}{2 \pi i} \oint_{0} \frac{d u}{2 \pi i}\left(-\frac{u}{1-w}+\frac{x}{w-z}\right)^{-t} \\
\cdot & (1+u) u^{t-1}(w-z)((w-1) w)^{-t} \Gamma(-t+1) \\
= & (z(1-z))^{-1 / 2}(1-t+(t-2) z+x t) \frac{\Gamma(1-t)\left(e^{-2 \pi i t}-1\right)}{(2-t)(1-t)}
\end{aligned}
$$


where the $w$-integration contour may be taken to be the unit circle.

It can be verified that the expressions obtained in the two examples satisfy the $\mathrm{KZ}$ equation.

We have also verified that these correlators respect the interesting conjecture made in ref. [1] that in the limit $x \rightarrow z$ they reduce to minimal model correlators. We hope to come back elsewhere and give a complete discussion of the validity of this conjecture based on our full integral representations.

\section{Proof of the Knizhnik-Zamolodchikov equations}

One may wonder whether the rules for contractions we have put forward, really reproduce the structure of the conformal theory. In order to settle this question in the affirmative we provide in this section an explicit proof that our $N$-point functions satisfy the KnizhnikZamolodchikov equations. In this proof we should not, therefore, make any use of the rules of contractions.

The Knizhnik-Zamolodchikov equation corresponding to the primary field at position $z_{m_{0}}$ may be written down as:

$$
\left\{t \partial_{z_{m_{0}}}+\sum_{m \neq m_{0}} \frac{2 D_{x_{m_{0}}}^{a} D_{x_{m}}^{a}}{z_{m}-z_{m_{0}}}\right\} W_{N}=0
$$

where $W_{N}$ is the $N$-point function after requisite integrals have been performed.

The structure of the proof is as follows:

For the selected position, $z_{m_{0}}$, of the primary field at that position, we shall define a function

$$
G(w)=\frac{1}{w-z_{m_{0}}}\left\{D_{x_{m_{0}}}^{+} G^{-}(w)+2 D_{x_{m_{0}}}^{3} G^{3}(w)+D_{x_{m_{0}}}^{-} G^{+}(w)\right\}
$$

where the $G^{a}(w)$ 's are functions to be defined and will turn out a posteriori to be

$$
G^{a}(w)=\left\langle J^{a}(w) \mathcal{O}\right\rangle
$$

where $\mathcal{O}$ is the collection of free field realizations of all our chiral vertex operators and screening charges. Indeed we shall evaluate the $G^{a}(w)^{\prime}$ 's using our contraction rules from that idea. However the point about the proof is that the function $G(w)$ eventually written down will only have pole singularities as a function of $w$, and will behave as $\mathcal{O}\left(w^{-2}\right)$ for $w \rightarrow \infty$, and thus the sum of residues will vanish. What we shall show explicitly is that the vanishing condition for this sum of residues is precisely the Knizhnik-Zamolodchikov equation on our $N$-point function. This should come as no surprise since this is merely the standard technique for proving the Knizhnik-Zamolodchikov equation. The point is that in the standard proof one makes use of associativity properties of the operators, and the purpose of our proof is exactly to establish that our rules for contractions in fact conform to those.

Now it is clear how we build the functions $G^{a}(w)$. It is useful to observe that to use

our contraction rules with the free field realizations eq.(3) is very easy in our correlator, 
since one may establish the rules:

$$
\begin{aligned}
\beta(w) & \rightarrow B(w) \\
\gamma(w) & \rightarrow-\sum_{i=1}^{M} \frac{D_{B_{i}}}{w-w_{i}} \\
-\sqrt{t / 2} \partial \varphi(w) & \rightarrow \sum_{i=1}^{M} \frac{k_{i}}{w-w_{i}}+\sum_{m=1}^{N-1} \frac{j_{m}}{w-z_{m}}
\end{aligned}
$$

where

$$
D_{B_{i}} \equiv \frac{\partial}{\partial B\left(w_{i}\right)}, \quad k_{i} \text { is defined as in section } 5
$$

Then (our notation here does not distinguish between integrands and integrated expressions)

$$
\begin{aligned}
G^{+}(w) & =B(w) W_{B} W_{N}^{\varphi} F \\
G^{3}(w) & =\left\{B(w) \sum_{i=1}^{M} \frac{D_{B_{i}}}{w-w_{i}}+\sum_{i=1}^{M} \frac{k_{i}}{w-w_{i}}+\sum_{m=1}^{N-1} \frac{j_{m}}{w-z_{m}}\right\} W_{B} W_{N}^{\varphi} F \\
G^{-}(w) & =\left\{-\sum_{i, j} B(w) \frac{D_{B_{i}} D_{B_{j}}}{\left(w-w_{i}\right)\left(w-w_{j}\right)}+(t-2) \sum_{i} \frac{D_{B_{i}}}{\left(w-w_{i}\right)^{2}}\right. \\
& \left.-2 \sum_{i, j} \frac{k_{i} D_{B_{j}}}{\left(w-w_{i}\right)\left(w-w_{j}\right)}-2 \sum_{m, j} \frac{j_{m} D_{B_{j}}}{\left(w-z_{m}\right)\left(w-w_{j}\right)}\right\} W_{B} W_{N}^{\varphi} F
\end{aligned}
$$

These expressions define our function $G(w)$ and from now on we may completely forget they came form applying our contraction rules to certain correlators.

In the following calculations the structure of the auxiliary $u$-integrations turns out to be very crucial. In fact, any dependence on an $x_{m}$ is via the combination $x_{m} / u_{m}$ so that we may write

$$
x_{m} \partial_{x_{m}}=-u_{m} \partial_{u_{m}}
$$

and subsequently do a partial integration in $u_{m}$, writing effectively

$$
x_{m} \partial_{x_{m}} W_{B} W_{N}^{\varphi} F \sim W_{B} W_{N}^{\varphi} \partial_{u_{m}}\left(u_{m} F\right)
$$

Let

$$
D_{x_{m_{0}}}^{-} G_{z_{m_{0}}}^{+}
$$

denote the contribution to the pole residue in $G(w)$ at $w=z_{m_{0}}$ coming from the term

$$
D_{x_{m_{0}}}^{-} G^{+}(w) \equiv D_{x_{m_{0}}}^{-} \oint_{z_{m_{0}}} \frac{d w}{2 \pi i} \frac{1}{w-z_{m_{0}}} G^{+}(w)
$$

in $G(w)$ etc. Then we find after some calculations for the pole at $z_{m_{0}}$ :

$$
D_{x_{m_{0}}}^{-} G_{z_{m_{0}}}^{+}=\sum_{\ell \neq m_{0}} \frac{x_{\ell} / u_{\ell}}{z_{m_{0}}-z_{\ell}} \partial_{x_{m_{0}}} W_{B} \cdot W_{N}^{\varphi} F
$$




$$
\begin{aligned}
2 D_{x_{m_{0}}}^{3} G_{z_{m_{0}}}^{3} & =2\left\{-\sum_{\ell \neq m_{0}} \frac{x_{\ell} / u_{\ell}}{z_{m_{0}}-z_{\ell}} u_{m_{0}} \partial_{x_{m_{0}}} W_{B} \cdot W_{N}^{\varphi}\right. \\
& \left.-\partial_{z_{m_{0}}} W_{B} \cdot W_{N}^{\varphi}+\frac{t}{2 j_{m_{0}}} W_{B} \cdot \partial_{z_{m_{0}}} W_{N}^{\varphi}\right\}\left(-j_{m_{0}}+u_{m_{0}}^{-1}\right) F \\
D_{x_{m_{0}}}^{+} G_{z_{m_{0}}}^{-} & =\left\{-\sum_{\ell \neq m_{0}} \frac{x_{\ell} / u_{\ell}}{z_{m_{0}}-z_{\ell}} \partial_{x_{m_{0}}} W_{B} \cdot W_{N}^{\varphi}\left[2 j_{m_{0}} u_{m_{0}}^{2 j_{m_{0}}}-u_{m_{0}}^{2 j_{m_{0}}-1}\right]\right. \\
& -2 \partial_{z_{m_{0}}} W_{B} \cdot W_{N}^{\varphi}\left[\left(2 j_{m_{0}}-1\right) u_{m_{0}}^{2 j_{m_{0}}-1}-u_{m_{0}}^{2 j_{m_{0}}-2}\right] \\
& +\left(t-2+2 j_{m_{0}}\right) \partial_{z_{m_{0}}} W_{B} \cdot W_{N}^{\varphi} u_{m_{0}}^{2 j_{m_{0}}-1} \\
& \left.+\frac{t}{j_{m_{0}}} W_{B} \cdot \partial_{z_{m_{0}}} W_{N}^{\varphi}\left[2 j_{m_{0}} u_{m_{0}}^{2 j_{m_{0}}-1}-u_{m_{0}}^{2 j_{m_{0}}-2}\right]\right\} \\
& \cdot e^{1 / u_{m_{0}}} \Gamma\left(2 j_{m_{0}}+1\right) \prod_{m \neq m_{0}} \Gamma\left(2 j_{m}+1\right) u_{m}^{2 j_{m}-1} e^{1 / u_{m}}
\end{aligned}
$$

This sums up to become

$$
t \partial_{z_{m_{0}}} W_{N}
$$

the first term in the Knizhnik-Zamolodchikov equation.

In a similar fashion the pole residue at $w=w_{j}$ turns out to give after some calculations (up to a factor independent of $w_{j}$ )

$$
\partial_{w_{j}}\left(\frac{t W_{B} \cdot W_{N}^{\varphi}}{B\left(w_{j}\right)\left(w_{j}-z_{m_{0}}\right)}\right)
$$

so that this term will vanish upon integration over $w_{j}$.

Finally the pole residues at the points $w=z_{m} \neq z_{m_{0}}$ rather easily give the remaining terms in the KZ equations, eq.(90) . Since there are no other singularities in $w$, the KZ equations have been proven.

\section{$7 \quad$ Projective invariance and global $S L(2)$ invariance}

In ref. [22 it was shown, that solutions of the Knizhnik-Zamolodchikov equations are projectively invariant provided the primary fields can add up to a singlet. In our case this is the requirement of global $S L(2)$ invariance. Thus, let us restrict to the case where the initial and final bra and ket carry just the vacuum and the dual vacuum: $j_{1}=0=j_{N}$, i.e. we are really looking at an $(N-2)$-point function. Then global $S L(2)$ invariance is the statement that

$$
\sum_{m=2}^{N-1} D_{x_{m}}^{a} W_{N-2}=0
$$

This is equivalent to the statement that in

$$
G^{a}(w)=\left\langle J^{a}(w) \mathcal{O}\right\rangle
$$

the leading behaviour as $w \rightarrow \infty$ is $\mathcal{O}\left(w^{-2}\right)$ rather than $\mathcal{O}\left(w^{-1}\right)$. From the expressions above, that is trivial for $G^{-}(w)$, and for $G^{3}(w)$ it follows from the fact that

$$
\sum_{i=1}^{M} k_{i}=-\sum_{m=1}^{N-1}\left(\rho_{m}-t \sigma_{m}\right)=-\sum_{m=1}^{N-1} j_{m}
$$


for $j_{N}=0$. For $G^{+}(w)$ it is more complicated to see. As previously discussed this is related to the fact that we are not using the projectively invariant vacuum, $\left\langle s l_{2}\right|$, in our calculations, rather we are using the dual vacuum, $\langle 0|$, for which $\langle 0| J_{0}^{+}=\langle 0| \beta_{0} \neq 0$.

What we are going to show is that the state,

$$
\langle 0| \beta_{0}
$$

even though it is non-vanishing, is BRST-exact in the sense of Felder [21], and that (hence) it decouples from correlators of BRST invariant operators.

First let us argue at the operator level, and subsequently at the level of our correlators. We write

$$
\langle 0| \beta_{0}=\langle 0| e^{-\sqrt{2 / t}} q_{\varphi} e^{\sqrt{2 / t} q_{\varphi}} \beta_{0}=\langle-1| \oint \frac{d z}{2 \pi i} e^{\sqrt{2 / t} \varphi(z)} \beta(z)
$$

where the bra state, $\langle-1|$, is the lowest weight state, $\langle j=-1|$. Now this integral is in fact the appropriate BRST operator in Felder's formulation. To see this, recall, that acting on the Fock space pertaining to

$$
j_{r, s}
$$

and labelled $F_{r, s}$, the relevant BRST operator (for which the BRST current is single valued) is

$$
Q_{r} \sim \oint \frac{d v_{0}}{2 \pi i} \ldots \frac{d v_{r-1}}{2 \pi i} S_{1}\left(v_{0}\right) \ldots S_{1}\left(v_{r-1}\right)
$$

with

$$
Q_{r}: F_{r, s} \mapsto F_{-r, s}
$$

For $j_{N}=0$, this is the Fock space with $r=1, s=0$, so that in fact

$$
2 j_{N}+1=1-0 \cdot t
$$

therefore the relevant BRST operator on this space is $Q_{1}$ which is just the one we obtained.

Next let us see how the argument works at the level of the correlator. We are going to show that inserting the operator

$$
\beta_{0}=\oint_{\infty} \frac{d w}{2 \pi i} \beta(w)
$$

furthest to the left in a correlator with $j_{N}=0$, is equivalent to inserting the BRST-charge operator

$$
\oint_{\infty} \frac{d w}{2 \pi i} \beta(w) e^{\sqrt{2 / t} \varphi(w)}
$$

furthest to the left of the operators and to the right of the bra $\langle-1|$.

Indeed in the first case, using the rules for building correlators, we obtain (up to normalization)

$$
\begin{aligned}
& \oint_{\infty} \frac{d w}{2 \pi i} B(w) \prod_{i} B\left(w_{i}\right)^{-k_{i}} \prod_{i<j}\left(w_{i}-w_{j}\right)^{2 k_{i} k_{j} / t} \\
& \cdot \prod_{n<m}\left(z_{n}-z_{m}\right)^{2 j_{n} j_{m} / t} \prod_{i, n}\left(w_{i}-z_{n}\right)^{2 k_{i} j_{n} / t} \prod_{n} u_{n}^{2 j_{n}-1} e^{1 / u_{n}}
\end{aligned}
$$


In the second case, we notice that the state labelled $\langle-1|$ is exactly a lowest weight state, $\left\langle j_{N}=-1\right|$, and we are formally looking at an $(N-1)$ point function with $j_{N}=-1$. Since we have

$$
\iota_{N-2}+j_{N-1}-j_{N}=\rho_{N-1}-\sigma_{N-1} t
$$

we see that the value of $\rho_{N-1}$ will be one unit larger in the case $j_{N}=-1$ than in the case $j_{N}=0$. This means we have one extra screening charge of the first kind in that case, which we may lift off the intertwining field furthest to the left, in other words that calculation will just be the one we seek to carry out. Using the rules developed we find in that case, letting $w$ denoting the position of the extra screening operator compared to the previous case (so in the formulas below, the index, $i$, runs over exactly the same set as before):

$$
\begin{aligned}
& \oint_{\infty} \frac{d w}{2 \pi i} B(w) \prod_{i} B\left(w_{i}\right)^{-k_{i}} \prod_{i}\left(w-w_{i}\right)^{-2 k_{i} / t} \prod_{n}\left(w-z_{n}\right)^{-2 j_{n} / t} \prod_{i<j}\left(w_{i}-w_{j}\right)^{2 k_{i} k_{j} / t} \\
\cdot & \prod_{n<m}\left(z_{n}-z_{m}\right)^{2 j_{n} j_{m} / t} \prod_{i, n}\left(w_{i}-z_{n}\right)^{2 k_{i} j_{n} / t} \prod_{n} u_{n}^{2 j_{n}-1} e^{1 / u_{n}} \\
= & \oint_{\infty} \frac{d w}{2 \pi i} B(w) \prod_{i} B\left(w_{i}\right)^{-k_{i}} \prod_{i}\left(1-w_{i} / w\right)^{-2 k_{i} / t} \prod_{n}\left(1-z_{n} / w\right)^{-2 j_{n} / t} w^{-2\left(\sum_{i} k_{i}+\sum_{n} j_{n}\right) / t} \\
\cdot & \prod_{i<j}\left(w_{i}-w_{j}\right)^{2 k_{i} k_{j} / t} \prod_{n<m}\left(z_{n}-z_{m}\right)^{2 j_{n} j_{m} / t} \prod_{i, n}\left(w_{i}-z_{n}\right)^{2 k_{i} j_{n} / t} \prod_{n} u_{n}^{2 j_{n}-1} e^{1 / u_{n}}
\end{aligned}
$$

We now use that

$$
-\sum_{i} k_{i}=\sum_{n} \rho_{n}-t \sum_{n} \sigma_{n}=\sum_{n} j_{n}-j_{N}
$$

Since we use the notation that sums over $i$ and $n$ are pertaining to the case with $j_{N}=0$, we see that the extra power of $w$ becomes zero, and for a very large integration contour for $w$, all $w$-dependence drops out except for the one in $B(w)$ so that we exactly prove the identity of the two cases also at the level of our correlators.

Having come this far, we may move the Felder-type BRST operator through all intertwining fields in exactly the same way as for minimal models, until in the end it hits the vacuum. Since we are only using screening operators of the first kind in the BRST operators, the procedure will work just as for the minimal models.

In addition it is rather easy to verify that the functions $G^{a}(w)$ have the expected pole residues for $\left\langle J^{a}(w) \mathcal{O}\right\rangle$ at points $w=z_{m}$. Further one verifies that there are no pole residues in $G^{+}(w)$ and $G^{3}(w)$ for $w=w_{i}$ the position of a screening charge. For $G^{-}(w)$ that residue is proportional to the total derivative

$$
\partial_{w_{i}}\left(D_{B_{i}} W_{B} \cdot W_{N}^{\varphi}\right)
$$

All of those remarks establish that our $N$-point blocks have the correct projective and global $S L(2)$ invariance properties. In particular, it gives rise to alternative representations for the $N$-point blocks, ones obtained by taking initial and final states to be vacua. This new representation is more symmetric and does not involve dual states [5], but involves a larger number of screening integrations in general. 


\section{Conclusions}

In this paper we have shown how to deal with fractional powers of free fields, and thereby we have managed to make sense of the second screening charge proposed by Bershadsky and Ooguri 15] for affine $S L(2)$ WZNW models. This has enabled us to build the most general conformal blocks for such theories on the sphere, even in the case of admissible representations with fractional levels. The ensuing integral formulas are tractable and have allowed us to verify many formal properties, such as projective invariance, global $S L(2)$ invariance, the fact that the conformal blocks satisfy the Knizhnik-Zamolodchikov equations etc. This gives hope that the relations to non perturbative string theory and topological field theory along the lines of [18, 19] may be made more explicit. Also our technique seems straightforward to generalize to higher groups and supergroups so that further progress in non perturbative string theory conceivably could be obtained.

\section{A Fractional Calculus}

Here we very briefly introduce fractional calculus [16]. For an analytic function, $f(z)$, the fractional derivative, $\partial_{z}^{a}$, is defined for any complex number, $a$. It satisfies the following axioms:

1. If $f(z)$ is an analytic function, the fractional derivative, $\partial^{\delta} f(z)$, is an analytic function of $z$ and $\delta$.

2. For $\delta$ integer, the result must agree with ordinary differentiation ( $\delta$ positive) or integration ( $\delta$ negative). By default the integration constants are put to zero, so that the function together with a maximum number of derivatives vanish at some point, like $z=0$.

3. $\delta=0$ is the identity.

4. Fractional differentiation is linear.

5. For fractional integration, $\Re \alpha>0, \Re \beta>0$

$$
\partial^{-\alpha} \partial^{-\beta} f(z)=\partial^{-(\alpha+\beta)} f(z)
$$

For $\Re a>0, \partial^{-a}$ satisfying the above is given by the Riemann-Liouville operator

$$
\partial^{-a} f(z)=\frac{1}{\Gamma(a)} \int_{0}^{z}(z-t)^{a-1} f(t) d t
$$

For any $a, b$

$$
\partial^{a} \partial^{b}=\partial^{a+b}
$$

Thus fractional differentiation is also defined. We principally need the rule, which is now easy to derive

$$
\partial_{x}^{a} x^{b}=\frac{\Gamma(b+1)}{\Gamma(b-a+1)} x^{b-a}
$$

(Notice that $\partial_{x}^{a} 1 \neq 0$ for $a$ not positive integer $(!)$ ). 


\section{B Example of consistency conditions for Wick con- tractions}

In this appendix we illustrate the non-trivial nature of the workings of the Wick contractions we have proposed. In fact, consider the reduction of

$$
\beta^{a}(z) \gamma^{a}\left(z^{\prime}\right)=\frac{\Gamma(a+1)}{\left(z-z^{\prime}\right)^{a}} \sum_{N}\left(\begin{array}{c}
a \\
N
\end{array}\right) \frac{1}{N !}\left(z-z^{\prime}\right)^{N}:\left(\beta(z) \gamma\left(z^{\prime}\right)\right)^{N}:
$$

where we have used the rules developed in sects. 2 and 3. Next consider the evaluation of

$$
I_{a, b}\left(z, z^{\prime}\right) \equiv\left(\beta^{a}(z) \gamma^{a}\left(z^{\prime}\right)\right)\left(\beta^{b}(z) \gamma^{b}\left(z^{\prime}\right)\right)
$$

This expression may be evaluated in two ways: either (i) by first using eq.(108) for both parentheses to reduce both of them to normal ordered products of integer powers of $\beta$ and $\gamma$, and then subsequently carrying out all remaining contractions, or else (ii) by simply using eq.(108) with $a+b$ replacing $a$. Obviously these two ways should lead to the same result for consistency. This requirement is part of the associativity properties for operators, and we consider them to be justified by the fact that our blocks satisfy the $\mathrm{KZ}$ equations. Here we demonstrate that the above condition gives rise to nontrivial identities for which we indicate an independent elementary proof.

The general contractions between integer powers of $\beta$ and $\gamma$ are carried out using the following trick:

$$
\begin{aligned}
\beta^{n}(z) \gamma^{m}\left(z^{\prime}\right) & =\left[\beta(z)+\left(z-z^{\prime}\right)^{-1} \partial_{\left.\gamma\left(z^{\prime}\right)\right]^{n} \gamma^{m}\left(z^{\prime}\right)}\right. \\
& =: \exp \left\{\left(z-z^{\prime}\right)^{-1} \partial_{\gamma\left(z^{\prime}\right)} \partial_{\beta(z)}\right\} \beta^{n}(z) \gamma^{m}\left(z^{\prime}\right): \\
& =\sum_{\ell} \frac{\left(z-z^{\prime}\right)^{-\ell}}{\ell !}: \partial_{\beta(z)}^{\ell} \beta^{n}(z) \partial_{\gamma\left(z^{\prime}\right)}^{\ell} \gamma^{m}\left(z^{\prime}\right): \\
& =\sum_{\ell} \frac{1}{\left(z-z^{\prime}\right)^{\ell} \ell !} \frac{\Gamma(n+1)}{\Gamma(n-\ell+1)} \frac{\Gamma(m+1)}{\Gamma(m-\ell+1)}: \beta^{n-\ell}(z) \gamma^{m-\ell}\left(z^{\prime}\right):
\end{aligned}
$$

Then one obtains after a few steps

$$
\begin{aligned}
I_{a, b}\left(z, z^{\prime}\right)= & \frac{\Gamma(a+1) \Gamma(b+1)}{\left(z-z^{\prime}\right)^{a+b}} \sum_{m, n, k, \ell}\left(\begin{array}{c}
a \\
m
\end{array}\right)\left(\begin{array}{c}
b \\
n
\end{array}\right)\left(\begin{array}{c}
m \\
k
\end{array}\right)\left(\begin{array}{c}
n \\
\ell
\end{array}\right) \\
& \cdot \frac{:\left(\beta(z) \gamma\left(z^{\prime}\right)\left(z-z^{\prime}\right)\right)^{m+n-k-\ell}:}{(m-\ell) !(n-k) !}
\end{aligned}
$$

On the other hand the second way of evaluation simply gives the result

$$
I_{a, b}\left(z, z^{\prime}\right)=\frac{\Gamma(a+b+1)}{\left(z-z^{\prime}\right)^{a+b}} \sum_{N}\left(\begin{array}{c}
a+b \\
N
\end{array}\right)\left(z-z^{\prime}\right)^{N} \frac{\left(\beta(z) \gamma\left(z^{\prime}\right)\right)^{N}:}{N !}
$$

Defining the generating functions

$$
\begin{aligned}
F_{a}(x) & =\sum_{N}\left(\begin{array}{c}
a \\
N
\end{array}\right) \frac{x^{N}}{N !} \\
G_{a b}(x) & =\sum_{m, n, k, \ell}\left(\begin{array}{c}
a \\
m
\end{array}\right)\left(\begin{array}{c}
b \\
n
\end{array}\right)\left(\begin{array}{c}
m \\
k
\end{array}\right)\left(\begin{array}{c}
n \\
\ell
\end{array}\right) \frac{x^{m+n-k-\ell}}{(m-\ell) !(n-k) !}
\end{aligned}
$$


we see that the consistency condition may be expressed as

$$
G_{a b}(x)=\left(\begin{array}{c}
a+b \\
b
\end{array}\right) F_{a+b}(x)
$$

We now briefly indicate how this identity may be proven. First we notice that we may write

$$
\left(\begin{array}{c}
a+b \\
a
\end{array}\right)=\left(\begin{array}{c}
a+b \\
b
\end{array}\right)=\oint \frac{d t}{2 \pi i} \frac{(1+t)^{a+b}}{t^{a+1}}
$$

where the contour may be taken as the unit circle, passing through the branch point at $t=-1$ of the integrand (for suitable values of the exponents). Then we write

$$
\begin{aligned}
G_{a b}(x) & =\sum_{p, q, k, \ell}\left(\begin{array}{c}
a \\
q+\ell
\end{array}\right)\left(\begin{array}{c}
b \\
p+k
\end{array}\right)\left(\begin{array}{c}
q+\ell \\
k
\end{array}\right)\left(\begin{array}{c}
p+k \\
\ell
\end{array}\right) \frac{x^{p+q}}{p ! q !} \\
& =\sum_{p, q, k, \ell} \prod_{i=1}^{4} \oint_{\mathcal{C}_{0}} \frac{d t_{i}}{2 \pi i} \frac{1}{t_{i}} \frac{\left(1+t_{1}\right)^{a}}{t_{1}^{q+\ell}} \frac{\left(1+t_{2}\right)^{b}}{t_{2}^{p+k}} \frac{\left(1+t_{3}\right)^{q+\ell}}{t_{3}^{k}} \frac{\left(1+t_{4}\right)^{p+k}}{t_{4}^{\ell}} \frac{x^{p+q}}{p ! q !}
\end{aligned}
$$

The identity is now obtained by successively doing 1 ) the sum over $\ell, 2$ ) the integral over $\left.t_{4}, 3\right)$ the sum over $\left.k, 4\right)$ the integral over $\left.t_{3}, 5\right)$ the integral over $t_{1}$ and $t_{2}$ in any order, and 6) the sum over $q$ with $p+q=N$. In each case one picks up residues after suitable deformations of the contours.

\section{Acknowledgement}

We are indebted to Anna Tollstén for discussions at the early stages of this work. Ming $\mathrm{Yu}$ would like to thank J.-B. Zuber and F.G. Malikov for private communications. M.Y. also thanks the Danish Research Academy for financial support.

\section{References}

[1] M. Wakimoto, Commun. Math. Phys. 104 (1986) 605

[2] D. Bernard and G. Felder, Commun. Math. Phys. 127 (1990) 145

[3] H. Awata, A. Tsuchiya and Y. Yamada, Nucl. Phys. B365 (1991) 680;

H. Awata, preprint KEK-TH-310/KEK Preprint 91-189

[4] P. Furlan, A.Ch. Ganchev, R. Paunov and V.B. Petkova, Nucl. Phys. B394 (1993) 665

[5] Vl.S. Dotsenko, Nucl. Phys. B338 (1990) 747

[6] V. Knizhnik and A. Zamolodchikov, Nucl. Phys. B247 (1984) 83

[7] V.A. Fateev and A.B. Zamolodchikov, Sov. J. Nucl. Phys. 43 (1986) 657

[8] K. Amoto, J. Math. Soc. Japan 39 (1987) 191 
[9] P. Christe and R. Flume, Nucl. Phys. B282 (1987) 466

[10] V.V. Schechtman and A.N. Varchenko, Invent. Math. 106 (1991) 139

[11] G. Felder and A. Varchenko, hep-th-9502165;

G. Felder and C. Wieczerkowski, hep-th-9411004

[12] D. Gepner and E. Witten, Nucl. Phys. B278 (1986) 493

[13] V.G. Kac and D.A. Kazhdan, Adv. Math. 34 (1979) 79

[14] F.G. Malikov, B.L. Feigin and D.B. Fuks, Funkt. Anal. Prilozhen 20 (1986) 25

[15] M. Bershadsky and H. Ooguri, Commun. Math. Phys. 126 (1989) 49

[16] A.C. McBride and G.F. Roach (eds.) Fractional Calculus (Pitman Advanced Publishing Program) (Boston 1985);

S.G. Samko, A.A. Kilbas and O.L. Marichec, Fractional Integrals and Derivatives, Gordon and Breach, Science Publishers (1993)

[17] V.G. Kac and M. Wakimoto, Proc. Natl. Acad. Sci. USA 85 (1988) 4956;

Adv. Ser. Math. Phys., Vol. 7 (World Scientific, 1989), p. 138

[18] H.-L. Hu and M. Yu, AS-ITP-92-32

[19] O. Aharony, O. Ganor, J. Sonnenschein and S. Yankielowicz, Nucl. Phys. B399 (1993) 527

[20] D. Friedan, E. Martinec and S. Shenker, Nucl. Phys. B271 (1986) 93

[21] G. Felder, Nucl. Phys. B317 (1989) 215 [Erratum: B324 (1989) 548]

[22] Vl.S. Dotsenko and V.A. Fateev, Nucl. Phys. B240[FS12] (1984) 312; Nucl. Phys. B251[FS13] (1985) 691

[23] H. Awata and Y. Yamada, Mod. Phys. Lett. A7 (1992) 1185

[24] B.L. Feigin and F.G. Malikov, preprint, private communication (1995) 